\title{
ERRATUM
}

\section{Hypertension and the eye: changing perspectives}

S Chatterjee, S Chattopadhyay, M Hope-Ross and PL Lip

Correction to: Journal of Human Hypertension 2002; 16: 667-675. doi:10.1038/sj.jhh.1001472

Journal of Human Hypertension (2002) 16, 901. doi:10.1038/sj.jhh.1001522

In the above paper, an error has been identified in the spelling of an author's name. The correct spelling appears below:

S Chattopadhyay 